\title{
Representations of copointed Hopf algebras arising from the tetrahedron rack
}

\author{
Bárbara Pogorelsky · Cristian Vay
}

the date of receipt and acceptance should be inserted later

Keywords Copointed Hopf algebras - Representations of Hopf algebras · Liftings of Nichols algebras · Affine racks

Mathematics Subject Classification (2000) 16W30

Abstract We study the copointed Hopf algebras attached to the Nichols algebra of the affine rack $\operatorname{Aff}\left(\mathbb{F}_{4}, \omega\right)$, also known as tetrahedron rack, and the 2 -cocycle -1 . We investigate the so-called Verma modules and classify all the simple modules. We conclude that these algebras are of wild representation type and not quasitriangular, also we analyze when these are spherical.

\section{Introduction}

We work over an algebraically closed field $\mathbb{k}$ of characteristic zero. Let $G$ be a finite non-abelian group and let $\mathbb{k}^{G}$ denote the algebra of functions on $G$. A Hopf algebra with coradical isomorphic to $\mathbb{k}^{G}$ for some $G$ is called copointed. Nicolás Andruskiewitsch and the second author began the study of the copointed Hopf algebras by classifying those finite-dimensional with $G=\mathbb{S}_{3}$ in [AV1] and by analyzing the representation theory of them in [AV2].

Since $\mathbb{k}^{G}$ is a commutative semisimple algebra, the representation theory of a copointed Hopf algebra over $\mathbb{k}^{G}$ is studied in [AV2] by analogy with the representation theory of semisimple Lie algebras, with $\mathbb{k}^{G}$ playing the role of the Cartan subalgebra and the induced modules from the simple onedimensional $\mathbb{k}^{G}$-modules as Verma modules.

Bárbara Pogorelsky

Instituto de Matemática, Universidade Federal do Rio Grande do Sul, Av. Bento Goncalves 9500, Porto Alegre, RS, 91509-900, Brazil, E-mail: barbara.pogorelsky@ufrgs.br

Cristian Vay

FaMAF-CIEM (CONICET), Universidad Nacional de Córdoba, Medina Allende s/n, Ciudad Universitaria, 5000 Córdoba, República Argentina, E-mail: vay@famaf.unc.edu.ar. 
There are few examples of Nichols algebras of finite-dimension over nonabelian groups, see for instance [G2, HLV]. In particular, those arising from affine racks are only seven, including the tetrahedron rack. If $X$ is one of these affine racks, then all the liftings of the Nichols algebra $\mathcal{B}(-1, X)$ over $\mathbb{k}^{G}$ were classified in [GIV], where $G$ is any group admitting a principal YD-realization of $X$ with constant 2 -cocycle -1 . Also the liftings of $\mathcal{B}(X,-1)$ over the group algebra $\mathbb{k} G$ were classified in [GIV].

The notation used in the following is explained in Section 3. Let $G$ be a finite group and $V \in \mathbb{k}_{\mathbb{k}^{G}} \mathcal{Y} \mathcal{D}$ a faithful principal YD-realization of the tetrahedron rack with constant 2 -cocycle -1 . The Nichols algebra $\mathcal{B}(V)$ has dimension 72. The ideal of relations of $\mathcal{B}(V)$ is generated by four quadratic elements and only one of degree six called $z$. By [GIV], the liftings of $\mathcal{B}(V)$ over $\mathbb{k}^{G}$ are the copointed Hopf algebras $\left\{\mathcal{A}_{G, \lambda}\right\}_{\lambda \in \mathbb{k}}$, in which the quadratic relations of $\mathcal{B}(V)$ still hold and the 6 -degree relation $z=0$ deforms to $z=\lambda\left(1-\chi_{z}^{-1}\right) \in \mathbb{k}^{G}$.

The goal of this paper is to investigate the representation theory of the family $\left\{\mathcal{A}_{G, \lambda}\right\}_{\lambda \in \mathbb{k}}$ following the strategy of [AV2]. We conclude that there are essentially two kinds of Verma modules. Here is an account of our main results which apply to any group $G$ admitting a faithful principal YD-realization of the tetrahedron rack with constant 2-cocycle -1 :

- Let $g \in G$. If the element $z=\lambda\left(1-\chi_{z}^{-1}\right)$ annihilates the generator of the Verma modules $M_{g}$, then $M_{g}$ inherits a structure of $\mathcal{B}(V)$-module such that it is a free $\mathcal{B}(V)$-module of rank 1 , see Lemma 14 . Hence $M_{g}$ has a unique simple quotient of dimension 1 called $\mathbb{k}_{g}$.

- Otherwise $M_{g}$ is the direct sum of six 12-dimensional non isomorphic simple projective modules $L_{i}^{g}$, see Lemma 15. Tables 1-6 in the Appendix describe the simple modules $L_{i}^{g}$.

- We prove that $\mathcal{A}_{G, \lambda}$ is of wild representation type, Proposition 17.

- We give a necessary condition for a copointed Hopf algebra to be quasitriangular, Lemma 8. As a consequence $\mathcal{A}_{G, \lambda}$ is not quasitriangular, Proposition 12.

- We characterize those $\mathcal{A}_{G, \lambda}$ which are spherical Hopf algebras, see Proposition 18

The other copointed Hopf algebras classified in [GIV] are defined by similar relations to $\mathcal{A}_{G, \lambda}$, roughly speaking a set of quadratic ones and other single relation of bigger degree, but their dimension are much bigger than $\operatorname{dim} \mathcal{A}_{G, \lambda}=$ $72|G|$. To extend this work to the other copointed Hopf algebras in [GIV], a better understanding of the corresponding Nichols algebras is needed. We hope that our work will be useful for this purpose.

The paper is organized as follows. In Section 2 we analyze the representation theory of copointed Hopf algebras with emphasis in the weight spaces of the modules, we characterize the one-dimensional modules and describe the subalgebra corresponding to the homogeneous elements of degree $e \in G$. In Section 3, we present our main object of study: the algebras $\mathcal{B}(V)$ and $\mathcal{A}_{G, \lambda}$. In Section 4 we concentrate our attention on representations of the algebras $\left\{\mathcal{A}_{G, \lambda}\right\}_{\lambda \in \mathbb{k}}$. A description of the simple $\mathcal{A}_{G, \lambda}$-modules is in the Appendix. 
1.1 Conventions and notation

We set $\mathbb{k}^{*}=\mathbb{k} \backslash\{0\}$. If $X$ is a set, $\mathbb{k} X$ denotes the free vector space over $X$.

Let $A$ be a Hopf algebra. Then $\Delta, \varepsilon, \mathcal{S}$ denote respectively the comultiplication, the counit and the antipode. The group of group-like elements is $G(A)$. Let ${ }_{A}^{A} \mathcal{Y D}$ be the category of Yetter-Drinfeld modules over $A$. The Nichols algebra $\mathcal{B}(V)$ of $V \in{ }_{A}^{A} \mathcal{Y} \mathcal{D}$ is the graded quotient $T(V) / \mathcal{J}$ where $\mathcal{J}(V)$ is the largest Hopf ideal of $T(V)$ generated as an ideal by homogeneous elements of degree $\geq 2[$ AS, 2.1].

Let $\left\{A_{[n]}\right\}_{n \geq 0}$ denote the coradical filtration of $A$. Assume $A_{[0]}=H$ is a Hopf subalgebra. Let $\operatorname{gr} A$ be the graded Hopf algebra associated to the coradical filtration. Then $\operatorname{gr} A \simeq R \# H$ where $R \in{ }_{H}^{H} \mathcal{Y} \mathcal{D}$ is called the diagram of $A$ and $V=R_{[1]} \in{ }_{H}^{H} \mathcal{Y D}$ is the infinitesimal braiding [AS, Definition 1.15]. If $R=\mathcal{B}(V)$, then $A$ is said to be a lifting of $\mathcal{B}(V)$ (over $H$ ).

Recall that two idempotents $\mathbf{e}, \widetilde{\mathbf{e}} \in A$ are orthogonal if $\mathbf{e} \widetilde{\mathbf{e}}=0=\widetilde{\mathbf{e e}}$. An idempotent is primitive if it is not possible to express it as the sum of two nonzero orthogonal idempotents. A set $\left\{\mathbf{e}_{i}\right\}_{i \in I}$ of idempotents of $A$ is complete if $1=\sum_{i \in I} e_{i}$.

Assume $\operatorname{dim} A<\infty$. Then $A$ is a Frobenius algebra, see e. $g$. [FMoS, Lemma 1.5]. Let e be a primitive idempotent of $A$. Then $t o p(A \mathbf{e})=A \mathbf{e} / \operatorname{rad}(A \mathbf{e})$ and the socle $\operatorname{soc}(A \mathbf{e})$ of $A \mathbf{e}$ are simple modules [CR, Theorems 54.11 and $58.12]$. Moreover, $A \mathbf{e}$ is the injective hull of $\operatorname{soc}(A \mathbf{e})$ and the projective cover of $\operatorname{top}(A \mathbf{e})$, see $e . \quad g$. [CR, page 400 and Theorem 58.14]. We denote by $\operatorname{Irr} A$ a set of representative of simple $A$-modules.

\section{Representations of copointed Hopf algebras}

Let $G$ be a finite group, $\mathbb{k} G$ the group algebra and $\mathbb{k}^{G}$ the algebra of functions on $G$. Let $\{g: g \in G\}$ and $\left\{\delta_{g}: g \in G\right\}$ be the dual basis of $\mathbb{k} G$ and $\mathbb{k}^{G}$, respectively; $e$ denotes the identity element of $G$.

If $M$ is a $\mathbb{k}^{G}$-module, then $M[g]=\delta_{g} \cdot M$ is the isotypic component of weight $g \in G$. We denote by $\mathbb{k}_{g}$ the one-dimensional $\mathbb{k}^{G}$-module of weight $g$. We define

$$
M^{\times}=\oplus_{g \neq e} M[g] \text { and } \operatorname{Supp} M=\{g \in G: M[g] \neq 0\} .
$$

Let $A$ be a finite-dimensional copointed Hopf algebra over $\mathbb{k}^{G}$, i. e. its coradical is isomorphic to $\mathbb{k}^{G}$. We consider $A$ as a left $\mathbb{k}^{G}$-module via the left adjoint action

$$
\operatorname{ad} \delta_{t}(a)=\sum_{s \in G} \delta_{s} a \delta_{t^{-1} s} \quad \forall t \in G, a \in A
$$

By [AV1, Lemma 3.1], $A=\oplus_{g \in G} A[g]$ is a $G$-graded algebra and

$$
\delta_{t} a_{s}=a_{s} \delta_{s^{-1} t} \quad \forall a_{s} \in A[s], s, t \in G .
$$


If $M$ is an $A$-module, then $M$ is a $\mathbb{k}^{G}$-module by restriction. Hence

$$
A[g] \cdot M[h] \subseteq M[g h] \quad \forall g, h \in G \text { by }(1) .
$$

That is, $M$ is a $G$-graded $A$-module.

We denote by $A_{\mathbb{k}^{G}}=A$ as right $\mathbb{k}^{G}$-module via the right multiplication. Its isotypic components are $\left(A_{\mathbb{k}^{G}}\right)[g]=A \delta_{g}$ for all $g \in G$. Note that $A$ is a $\mathbb{k}^{G}$-bimodule with the above actions since $\mathbb{k}^{G} \subseteq A[e]$.

Let $R \in \mathbb{k}_{\mathbb{k}^{G}}^{G} \mathcal{Y} \mathcal{D}$ be the diagram of $A$. Then the multiplication in $A$ induces an isomorphism $R \otimes \mathbb{k}^{G} \longrightarrow A$ of $\mathbb{k}^{G}$-bimodules [AAGMV, Lemma 4.1]. Hence we can think of $R$ as a left $\mathbb{k}^{G}$-submodule of $A$ and therefore

$$
A[g]=R[g] \mathbb{k}^{G} \text { and }\left(A_{\mathbb{k}^{G}}\right)[g]=R \delta_{g} \quad \forall g \in G .
$$

As in [AV2], we define the Verma module of $A$ of weight $g \in G$ as the induced module

$$
M_{g}=\operatorname{Ind}_{\mathbb{k}^{G}}^{A} \mathbb{k}_{g}=A \otimes_{\mathbb{k}^{G}} \mathbb{k} \delta_{g} .
$$

Then $M_{g}$ is projective, being induced from a module over a semisimple algebra, and hence injective, because $A$ is Frobenius. By (1) and (3), the weight spaces satisfy $M_{g}[h]=R\left[h g^{-1}\right] \delta_{g}$ for all $h \in G$. Also, $M_{g}=A \delta_{g}=R \delta_{g}$ and $A=$ $\oplus_{g \in G} M_{g}$.

Notice that if $L$ is a simple $A$-module and $0 \neq v \in L[g]$, then $L$ is a quotient of $M_{g}$ via $\delta_{g} \mapsto \delta_{g} \cdot v=v$.

Let $\mathbf{e} \in A$ be an idempotent. We say that $\mathbf{e}$ is a $g$-idempotent if $\mathbf{e} \in R[e] \delta_{g}$. A set $\left\{\mathbf{e}_{i}\right\}_{i \in I}$ of $g$-idempotents is called complete if $\delta_{g}=\sum_{i \in I} \mathbf{e}_{i}$. Next lemma ensures that there always exists a complete set of orthogonal primitive $g$ idempotents.

Lemma 1. Let $g \in G$, e be a g-idempotent and $\mathcal{E}_{g}=\left\{\mathbf{e}_{i}\right\}_{i \in I}$ be a set of orthogonal idempotents of $A$ such that $\delta_{g}=\sum_{i \in I} \mathbf{e}_{i}$.

(a) $\mathcal{E}_{g}$ is a complete set of $g$-idempotents.

(b) $\mathbf{e}$ is primitive if and only if it is not possible to express $\mathbf{e}$ as a sum of orthogonal g-idempotents.

(c) There is a complete set of orthogonal primitive g-idempotents in $A$.

(d) $\mathbf{e} \cdot M=\mathbf{e} \cdot M[g] \subseteq M[g]$ for any A-module $M$.

(e) If $\# \mathcal{E}_{g}=\operatorname{dim} R[e]$, then $\mathbf{e}_{i}$ is primitive for all $i \in I$. Moreover, if $\mathbf{e}$ is primitive, then $\mathbf{e}=\mathbf{e}_{i}$ for some $i \in I$.

(f) If $\# \mathcal{E}_{g}=\operatorname{dim} R[e]$, then $A \mathbf{e}_{i} \not$ A $\mathbf{e}_{j}$ if $i \neq j$.

Proof (a) Fix $i \in I$ and set $\alpha=\mathbf{e}_{i}$ and $\beta=\sum_{i \neq j \in I} \mathbf{e}_{j}$. If $t \in G$ and $t \neq g$, then $0=\delta_{g} \delta_{t}=\alpha \delta_{t}+\beta \delta_{t}$. Since $\alpha$ and $\beta$ are orthogonal, $\alpha \delta_{t}=0$. Hence $\alpha=\alpha \delta_{g}$ because $1=\sum_{g \in G} \delta_{g}$. Similarly $\alpha=\delta_{g} \alpha$. Let $a_{s} \in R[s]$ such that $\alpha=\sum_{s \in G} a_{s} \delta_{g}$. Then $\alpha=\delta_{g} \alpha=\sum_{s \in G} \delta_{g} a_{s} \delta_{g}=\sum_{s \in G} a_{s} \delta_{s^{-1} g} \delta_{g}=a_{e} \delta_{g}$. That is, $\alpha=\mathbf{e}_{i}$ is a $g$-idempotent. 
(b) The first implication is obvious. For the second implication, we proceed as in (a). (c) follows from (a) and (b). (d) holds because $\mathbf{e} \in R[e] \delta_{g}$.

(e) is a consequence of the fact that $\mathcal{E}_{g}$ is a basis of $R[e] \delta_{g}$. Indeed, pick $\alpha=\mathbf{e}_{i} \in \mathcal{E}_{g}$ and suppose $\alpha=a+b$ with $a$ and $b$ orthogonal $g$-idempotents of $A$. Then $(A a)[e] \oplus(A b)[e]=(A \alpha)[e]=\left(\mathbb{k} \mathcal{E}_{g}\right) \alpha=\mathbb{k} \alpha$ and therefore $a=0$ or $b=0$. For the second statement, we write $\mathbf{e}=\sum_{i \in I} a_{i} \mathbf{e}_{i}$ with $a_{i} \in \mathbb{k}, i \in I$. Since $\mathbf{e}^{2}=\mathbf{e}, a_{i}=0$ or 1 for all $i \in I$ and hence $\mathbf{e}=\mathbf{e}_{i}$ for some $i \in I$.

(f) $\left(A \mathbf{e}_{i}\right)[e]=\mathbb{k} \mathbf{e}_{i} \neq\left(A \mathbf{e}_{j}\right)[e]=\mathbb{k} \mathbf{e}_{j}$ if $i \neq j$. Hence $A \mathbf{e}_{i} \neq A \mathbf{e}_{j}$.

Given a set of idempotents $\mathcal{E}$ and an $A$-module $M$, we write

$$
\operatorname{Supp}_{\mathcal{E}} M=\{\mathbf{e} \in \mathcal{E}: \mathbf{e} \cdot M \neq 0\} .
$$

By [CR, Theorem 54.16] if $L$ is a simple $A$-module and e $\in \operatorname{Supp}_{\mathcal{E}} L$, then

$$
\operatorname{top}(A \mathbf{e}) \simeq L .
$$

This allows us to analyze the dimension of the weight spaces of the simple $A$-modules using $g$-idempotents.

Lemma 2. Let $g \in G$ and $\mathcal{E}_{g}=\left\{\mathbf{e}_{i}\right\}_{i \in I}$ be a complete set of orthogonal primitive g-idempotents. Let $L$ be a simple A-module.

(a) $\operatorname{dim} L[g]=\# \operatorname{Supp}_{\mathcal{E}_{g}} L$.

(b) If $\# \mathcal{E}_{g}=\operatorname{dim} R[e]$ or 1 , then $\operatorname{dim} L[g]=1$ or 0 .

(c) $\mathcal{E}_{g}=\bigcup_{L \in \operatorname{Irr} A} \operatorname{Supp}_{\mathcal{E}_{g}} L$ is a partition.

(d) $\operatorname{dim} R[e] \geq \sum_{L \in \operatorname{Irr} A}(\operatorname{dim} L[g])^{2}=\sum_{L \in \operatorname{Irr} A}\left(\# \operatorname{Supp}_{\mathcal{E}_{g}} L\right)^{2} \geq \# \mathcal{E}_{g}$.

Proof (a) By [CR, Theorem 54.16], $\operatorname{dim} \mathbf{e}_{i} \cdot L=1$ for all $\mathbf{e}_{i} \in \operatorname{Supp}_{\mathcal{E}_{g}} L$. Pick $w_{i} \in \mathbf{e}_{i} \cdot L-\{0\}$ for each $i \in I$. Then $\left\{w_{i}: i \in I\right\}$ is a basis of $L[g]$ since $v=\delta_{g} \cdot v=\sum_{\mathbf{e}_{i} \in \operatorname{Supp}_{\mathcal{E}_{g}} L} \mathbf{e}_{i} \cdot v$ for all $v \in L[g]$.

(b) If $\# \mathcal{E}_{g}=1$, then $\operatorname{dim} L[g]=1$ or 0 by (a). If $\# \mathcal{E}_{g}=\operatorname{dim} R[e]$, the statement follows from (a) and Lemma 1 (f).

(c) is clear. (d) follows from (a) and (c) since

$$
R[e] \delta_{g}=\oplus_{i \in I} R[e] \mathbf{e}_{i}=\oplus_{L \in \operatorname{Irr} A} \bigoplus_{\mathbf{e}_{i} \in \operatorname{Supp}_{\mathcal{E}_{g}} L} R[e] \mathbf{e}_{i}
$$

In some cases, the simple $A$-modules can be distinguished by their weight spaces.

Lemma 3. Let $g \in G$ and $\mathcal{E}_{g}=\left\{\mathbf{e}_{i}\right\}_{i \in I}$ be a complete set of orthogonal primitive g-idempotents and assume that top $\left(A \mathbf{e}_{i}\right)$ and top $\left(A \mathbf{e}_{j}\right)$ are not isomorphic as $\mathbb{k}^{G}$-modules for all $i \neq j$. Let $L$ be a simple $A$-module. Then $L \simeq \operatorname{top}\left(A \mathbf{e}_{i}\right)$ as $A$-modules if and only if $L \simeq \operatorname{top}\left(A \mathbf{e}_{i}\right)$ as $\mathbb{k}^{G}$-modules.

Proof If $L \simeq \operatorname{top}\left(A \mathbf{e}_{i}\right)$ as $\mathbb{k}^{G}$-modules, then $g \in \operatorname{Supp} L$. Hence $L \simeq \operatorname{top}\left(A \mathbf{e}_{j}\right)$ for some $j$. Then $i=j$ because $\operatorname{top}\left(A \mathbf{e}_{i}\right)$ and $\operatorname{top}\left(A \mathbf{e}_{j}\right)$ are not isomorphic as $\mathbb{k}^{G}$-modules for $i \neq j$. The other implication is obvious. 
For each $g \in G$, let $\mathcal{E}_{g}$ be a complete set of orthogonal primitive $g$ idempotents. If $\mathbf{e}, \tilde{\mathbf{e}} \in \mathcal{E}_{g}$ and $\mathbf{e} A \tilde{\mathbf{e}} \neq 0$, it is said that $\mathbf{e}$ and $\tilde{\mathbf{e}}$ are linked. This is an equivalence relation [CR, Definition 55.1]. Let $\mathcal{E}_{g}=\bigcup_{i \in I_{g}} B_{i}$ be the corresponding partition. The subalgebra $A[e]=R[e] \mathbb{K}^{G}$ can be used to compute the simple $A$-modules, see for instance [NaVO, Theorem 2.7.2].

Lemma 4. Let $\mathcal{E}_{g}=\bigcup_{i \in I_{g}} B_{i}$ be as above. Then $\bigoplus_{\mathbf{e} \in B_{i}} A[e] \mathbf{e}$ is a subalgebra such that

$$
\left\{L[g]: L \in \operatorname{Irr} A \text { and } B_{i} \cap \operatorname{Supp}_{\mathcal{E}_{g}} L \neq \emptyset\right\}
$$

is a set of representative simple modules. Moreover as algebras

$$
A[e]=\prod_{g \in G, i \in I_{g}} \bigoplus_{\mathbf{e} \in B_{i}} A[e] \mathbf{e}
$$

Proof By (1), ẽ $=0=\tilde{\mathbf{e} e}$ if either $\mathbf{e} \in \mathcal{E}_{g}$ and $\tilde{\mathbf{e}} \in \mathcal{E}_{h}$ with $g \neq h$ or $\mathbf{e}, \tilde{\mathbf{e}} \in$ $\mathcal{E}_{g}$ but are not linked. Clearly, $B_{i}$ is a complete set of orthogonal primitive idempotents of $\bigoplus_{\mathbf{e} \in B_{i}} A[e] \mathbf{e}$. Also $\operatorname{top}(A[e] \mathbf{e})=L[g]$ since $L[g]=\operatorname{top}(A \mathbf{e})[g]=$ $\overline{A[e] \mathbf{e}}$ for all $\mathbf{e} \in \mathcal{E}_{g}$.

For $g \in G$, we define the linear map $\chi_{g}: A \mapsto \mathbb{k}$ by

$$
\chi_{g}(r f)=\varepsilon(r) f(g) \quad \forall r f \in A=R \mathbb{k}^{G} .
$$

If $\chi_{g}$ is an algebra map, then $\mathbb{k}_{g}$ is also an $A$-module. Notice that Nichols algebras satisfy the hypothesis of the next lemma by [AV1, Lemma 3.1 (f)].

Lemma 5. Let $G$ be a finite group, $A$ a finite-dimensional copointed Hopf algebra over $\mathbb{k}^{G}$ with diagram $R \in \mathbb{k}_{\mathbb{k}^{G}} \mathcal{Y} \mathcal{D}$ and $\chi: A \mapsto \mathbb{k}$ an algebra map. If $R$ is generated by $R^{\times}$as an algebra, then $\chi=\chi_{g}$ for some $g \in G$ and $G\left(A^{*}\right)$ is a subgroup of $G$ via $\chi_{g} \mapsto g$.

Proof Let $g \in G$ such that $\chi(f)=f(g)$ for all $f \in \mathbb{k}^{G}$. By (1), $\chi\left(R^{\times}\right)=0$ and then $\chi=\chi_{g}$. Since $\chi_{g} * \chi_{h}$ is an algebra map and $\chi_{g} * \chi_{h}(f)=f(g h)$ for all $f \in \mathbb{k}^{G}$, the proposition follows.

Example 1 Let $V \in \mathbb{k}_{\mathbb{k}^{G}}^{G} \mathcal{Y} \mathcal{D}$ with finite-dimensional Nichols algebra $\mathcal{B}(V)$. Then $\left\{\delta_{g}: g \in G\right\}$ is a complete set of orthogonal primitive idempotents of $\mathcal{B}(V) \# \mathbb{k}^{G}$ and therefore $\left\{\mathbb{k}_{g}: g \in G\right\}$ are its simple modules.

Let $\int_{A}^{r}$ (resp. $\int_{A}^{l}$ ) denote the space of right (resp. left) integrals, see for example [Mo]. If $t \in \int_{A}^{r}$, then $\alpha \in G\left(A^{*}\right)$ is said to be distinguished whether $a t=\alpha(a) t$ for all $a \in A$.

Lemma 6. Let $G$ be a finite group, $A$ a finite-dimensional copointed Hopf algebra over $\mathbb{k}^{G}$ and $\alpha=\chi_{g} \in G\left(A^{*}\right)$ the distinguished group-like element. If e $i s$ a primitive idempotent, then

$$
\operatorname{Supp}(\operatorname{top}(A \mathbf{e}))=g^{-1} \operatorname{Supp}(\operatorname{soc}(A \mathbf{e})) .
$$

In particular, $\int_{A}^{l}=\operatorname{soc}\left(A \mathbf{e}_{g^{-1}}\right) \subset R[g] \mathbf{e}_{g^{-1}}$ where $\mathbf{e}_{g^{-1}}$ is the primitive $g^{-1}$ idempotent such that $\operatorname{top}\left(A \mathbf{e}_{g^{-1}}\right) \simeq \mathbb{k}_{g^{-1}}$. 
Proof Let $\eta: A \rightarrow A$ be the Nakayama automorphism. If $M$ is an $A$-module, then $\bar{M}$ denotes the vector space $M$ with action $a \cdot m=\eta^{-1}(a) m$ for all $a \in A$, $m \in M$. Since $\eta^{-1}(a)=\left\langle\alpha^{-1}, S^{2}(a)_{1}\right\rangle S^{2}(a)_{2}$ for all $a \in A$, see e. g. [FMoS, Lemma 1.5], $M\left[g^{-1} h\right]=\bar{M}[h]$ for all $h \in G$. Finally, $\operatorname{top}(A \mathbf{e})=\overline{\operatorname{soc}(A \mathbf{e})}$, see e. $g .[\mathrm{NeSc}$, Lemma 2], and the lemma follows.

We include the next lemma for completeness.

Lemma 7. Let $A$ be an algebra and $a_{1}, \ldots, a_{n}$ be idempotents of $A$ such that $a_{i} a_{j}=a_{j} a_{i}$ for all $i, j=1, \ldots, n$. Set

$$
\mathbf{e}_{i}=a_{i}+a_{i} \sum_{\ell=1}^{i-1}(-1)^{\ell} \sum_{1 \leq j_{1}<\cdots<j_{\ell} \leq i-1} a_{j_{1}} \cdots a_{j_{\ell}} .
$$

Then $\mathbf{e}_{i} \mathbf{e}_{j}=\delta_{j, i} \mathbf{e}_{i}$ for all $i, j=1, \ldots, n$.

Proof For $j<i$, we write

$$
\begin{aligned}
\mathbf{e}_{i}=a_{i}+a_{i} \sum_{\ell=1}^{i-1}(-1)^{\ell} \sum_{\substack{1 \leq j_{1}<\cdots<j_{\ell} \leq i-1 \\
j_{s} \neq j}} a_{j_{1}} \cdots a_{j_{\ell}} \\
+a_{i} \sum_{\ell=1}^{i-1}(-1)^{\ell} \sum_{\substack { \ell \\
\begin{subarray}{c}{1 \leq j_{1}<\cdots<j_{\ell} \leq i-1 \\
j_{s}=j \text { for some } s{ \ell \\
\begin{subarray} { c } { 1 \leq j _ { 1 } < \cdots < j _ { \ell } \leq i - 1 \\
j _ { s } = j \text { for some } s } }\end{subarray}} a_{j_{1}} \cdots a_{j_{\ell}} .
\end{aligned}
$$

Then $a_{j} \mathbf{e}_{i}=0$ and hence $\mathbf{e}_{j} \mathbf{e}_{i}=\delta_{i, j} \mathbf{e}_{i}$ for all $i, j=1, \ldots, n$.

The order of the set $\left\{a_{i}\right\}$ alters the result of the above lemma. Moreover, it can produce $\mathbf{e}_{i}=0$ for some $i$. For example: $\{1, a\}$ and $\{a, 1\}$ with $a$ an idempotent.

\subsection{Quasitriangular copointed Hopf algebras}

Let $G$ be a non-abelian group and $A$ be a quasitriangular finite-dimensional copointed Hopf algebra over $\mathbb{k}^{G}$ with $R$-matrix $R \in A \otimes A$. Let $\left(A_{R}, R\right)$ be its unique minimal subquasitriangular Hopf algebra $[\mathrm{R}]$. Then $A_{R}=H B$ with $H, B \subseteq A$ Hopf subalgebras such that $B \simeq H^{* \text { cop }}$ by [R, Proposition 2 and Theorem 1].

Lemma 8. $H, B$ and $A_{R}$ are pointed Hopf algebras over abelian groups. Moreover, $A_{R}$ is neither a group algebra nor the bosonization of its diagram by $G\left(A_{R}\right)$.

Proof Since $H_{[0]}=H \cap A_{[0]}$ and $B_{[0]}=B \cap A_{[0]}$, there are group epimorphisms $G \rightarrow G_{H}$ and $G \rightarrow G_{B}$ such that $H_{[0]}=\mathbb{k}^{G_{H}}$ and $B_{[0]}=\mathbb{k}^{G_{B}}$. Then there is an epimorphism of Hopf algebras $B \stackrel{\simeq}{\longrightarrow} H^{* \text { cop }} \longrightarrow \mathbb{k} G_{H}$. By [Mo, Corollary 5.3.5], the restriction $B_{[0]}=\mathbb{k}^{G_{B}} \rightarrow \mathbb{k} G_{H}$ is surjective. Thus $G_{H}$ is an abelian 
group. Mutatis mutandi, we see that $G_{B}$ is also an abelian group. Hence $H$ and $B$ are generated by skew-primitives and group-likes elements by [An, Theorem 2] and therefore also is $A_{R}=H B$. Then $A_{R}=H B, H$ and $B$ are pointed Hopf algebras over abelian groups. Set $\Gamma=G\left(A_{R}\right)$.

Now we assume $A_{R}=\mathbb{k} \Gamma$ and let $\delta_{g} \in \mathbb{k}^{G} \backslash \mathbb{k} \Gamma$. By a property of the $R$-matrix, it must hold $R \Delta\left(\delta_{g}\right)=\Delta^{\operatorname{cop}}\left(\delta_{g}\right) R$. However, this is not possible since $R$ is invertible and $\mathbb{k}^{G}$ is commutative but not cocommutative. Then $A_{R} \neq \mathbb{k} \Gamma$.

Finally, we assume that $A_{R}=\mathcal{B}(V) \# \mathbb{k} \Gamma$ where $\mathcal{B}(V)$ is the diagram of $A_{R}$ which is a Nichols algebra by [An, Theorem 2]. Let $R_{0} \in \mathbb{k} \Gamma \otimes \mathbb{k} \Gamma$ and $R^{+} \in \mathcal{B}(V)^{+} \# \mathbb{k} \Gamma \otimes \mathbb{k} \Gamma+\mathbb{k} \Gamma \otimes \mathcal{B}(V)^{+} \# \mathbb{k} \Gamma$ such that $R=R_{0}+R^{+}$. Then $R_{0}$ is invertible since $R$ is so and $\mathcal{B}(V)^{+}$is nilpotent. If $\delta_{g} \in \mathbb{k}^{G} \backslash \mathbb{k} \Gamma$, then it must hold $R_{0} \Delta\left(\delta_{g}\right)=\Delta^{c o p}\left(\delta_{g}\right) R_{0}$ by a property of the $R$-matrix. As above, this is not possible. Therefore $A_{R} \neq \mathcal{B}(V) \# \mathbb{k} \Gamma$.

\section{The affine rack $\operatorname{Aff}\left(\mathbb{F}_{4}, \omega\right)$ and their associated algebras}

Let $\mathbb{F}_{4}$ be the finite field of four elements and $\omega \in \mathbb{F}_{4}$ such that $\omega^{2}+\omega+1=0$. The affine rack $\operatorname{Aff}\left(\mathbb{F}_{4}, \omega\right)$ is the set $\mathbb{F}_{4}$ with operation $a \triangleright b=\omega b+\omega^{2} a$.

Let $\left(\cdot, g, \chi_{G}\right)$ be a faithful principal YD-realization of $\left(\mathrm{Aff}\left(\mathbb{F}_{4}, \omega\right),-1\right)$ over a finite group $G$ [AG3, Definition 3.2], that is

- . is an action of $G$ over $\mathbb{F}_{4}$,

$-g: \mathbb{F}_{4} \rightarrow G$ is an injective function such that $g_{h \cdot i}=h g_{i} h^{-1}$ and $g_{i} \cdot j=i \triangleright j$ for all $i, j \in \mathbb{F}_{4}, h \in G$

- $\chi_{G}: G \rightarrow \mathbb{k}^{*}$ is a multiplicative character such that $\chi_{G}\left(g_{i}\right)=-1$ for all $i \in \mathbb{F}_{4}$; we can consider such a $\chi_{G}$ by [AG3, Lemma $\left.3.3(\mathrm{~d})\right]$.

These data define a structure on $V=\mathbb{k}\left\{x_{i}\right\}_{i \in \mathbb{F}_{4}}$ of Yetter-Drinfeld module over $\mathbb{k}^{G}$ via

$$
\delta_{t} \cdot x_{i}=\delta_{t, g_{i}^{-1}} x_{i} \quad \text { and } \quad \lambda\left(x_{i}\right)=\sum_{t \in G} \chi_{G}\left(t^{-1}\right) \delta_{t} \otimes x_{t^{-1} \cdot i} \forall t \in G, i \in X .
$$

We obtain (5) using the fact that the categories ${ }_{\mathbb{k}^{G}}^{\mathbb{k}^{G}} \mathcal{Y} \mathcal{D}$ and $\mathbb{k}_{\mathbb{k}}^{\mathbb{k} G} \mathcal{Y} \mathcal{D}$ are braided equivalent [AG1, Proposition 2.2.1], see [GIV, Subsection 3.2] for details.

We denote by $G^{\prime}$ the subgroup of $G$ generated by $\left\{g_{i}\right\}_{i \in \mathbb{F}_{4}}$. Then $G^{\prime}$ is a quotient of the enveloping group of $\operatorname{Aff}\left(\mathbb{F}_{4}, \omega\right)[\mathrm{EG}, \mathrm{J}]$ :

$$
G_{\mathrm{Aff}\left(\mathbb{F}_{4}, \omega\right)}=\left\langle g_{i} \mid g_{i} g_{j}=g_{i \triangleright j} g_{i}, i, j \in \mathbb{F}_{4}\right\rangle \text {. }
$$

Let $m \in \mathbb{N}$. We denote by $C_{m}=\langle t\rangle$ the cyclic group of order $m$. The semidirect product group $\mathbb{F}_{4} \rtimes_{\omega} C_{6 m}$ is given by $t \cdot i=\omega i$ for all $i \in \mathbb{F}_{4}$.

Examples 9. (1) Let $k, m \in \mathbb{N}, 0 \leq k<m$. The $(m, k)$-affine realization of (Aff $\left.\left(\mathbb{F}_{4}, \omega\right),-1\right)$ over $\mathbb{F}_{4} \rtimes_{\omega} C_{6 m}[\mathrm{GIV}$, Proposition 2.6] is defined by

$-g: \mathbb{F}_{4} \rightarrow \mathbb{F}_{4} \rtimes_{\omega} C_{6 m}, i \mapsto g_{i}=\left(i, t^{6 k+1}\right) ;$ 
- $: \mathbb{F}_{4} \rtimes_{\omega} C_{6 m} \rightarrow \mathbb{F}_{4}$ is $h \cdot i=j$, if $h g_{i} h^{-1}=g_{j}$;

$-\chi_{\mathbb{F}_{4} \rtimes_{\omega} C_{6 m}}: \mathbb{F}_{4} \rtimes_{\omega} C_{6 m} \longmapsto \mathbb{k}^{*},\left(j, t^{s}\right) \mapsto(-1)^{s}, \forall i, j \in A, s \in \mathbb{N}$.

(2) The next example gives a nontrivial lifting of $\mathcal{B}(V)$, see the next subsection. Suppose that $m \mid 6 k+1$. Let $G_{1}$ be a finite group with a multiplicative character $\chi_{G_{1}}: G_{1} \rightarrow \mathbb{k}^{*}$ such that $\chi_{G_{1}}^{6} \neq 1$. Then the $(m, k)$-affine realization is extended to a principal YD-realization over $G=\mathbb{F}_{4} \rtimes_{\omega} C_{6 m} \times G_{1}$ setting $G_{1} \cdot i=i$ and $\chi_{G}=\chi_{\mathbb{F}_{4} \rtimes_{\omega} C_{6 m}} \times \chi_{G_{1}}$. Note that $z \in T(V)[e]$ and $\chi_{G}^{6} \neq 1$, where $z$ is defined in (7).

(3) Let $\left(\cdot, g, \chi_{G}\right)$ be a faithful principal YD-realization of $\left(\operatorname{Aff}\left(\mathbb{F}_{4}, \omega\right),-1\right)$ over a finite group $G$. If $G^{\prime} \leq G_{1} \leq G$ are subgroups, then $\left(\cdot, g,\left(\chi_{G}\right)_{\mid G_{1}}\right)$ is a faithful principal YD-realization of $\left(\operatorname{Aff}\left(\mathbb{F}_{4}, \omega\right),-1\right)$ over $G_{1}$. For instance, $G_{1}=\operatorname{ker} \chi_{G}^{6}$.

\subsection{A Nichols algebra over $\operatorname{Aff}\left(\mathbb{F}_{4}, \omega\right)$}

From now on, we fix a faithful principal YD-realization $\left(\cdot, g, \chi_{G}\right)$ over a finite group $G$ of $\left(\operatorname{Aff}\left(\mathbb{F}_{4}, \omega\right),-1\right)$. Let $V \in \in_{\mathbb{k}^{G}}^{\mathbb{k}^{G}} \mathcal{Y D}$ be as in (5).

In [GIV, Subsection 2.2] it was discussed how braided functors modify the Nichols algebras. As a consequence the defining relations of the Nichols algebra $\mathcal{B}(V)$ were calculated [GIV, Proposition 2.10 (b)] using previous results of [G1] for the pointed case.

Namely, $\mathcal{B}(V)$ is the quotient of $T(V)$ by the ideal $\mathcal{J}(V)$ generated by

$$
\begin{aligned}
& x_{i}^{2}, \quad x_{j} x_{i}+x_{i} x_{(\omega+1) i+\omega j}+x_{(\omega+1) i+\omega j} x_{j} \quad \forall i, j \in \mathbb{F}_{4} \text { and } \\
& z:=\left(x_{\omega} x_{0} x_{1}\right)^{2}+\left(x_{1} x_{\omega} x_{0}\right)^{2}+\left(x_{0} x_{1} x_{\omega}\right)^{2} .
\end{aligned}
$$

We are specially interested in the case where $z \in T(V)[e]$, since otherwise the liftings of $\mathcal{B}(V)$ are trivial, see Theorem 11 (b). In Example 9 (2) this condition is satisfied.

Let $\mathbb{B}$ be the basis of $\mathcal{B}(V)$ consisting of all possible words $m_{1} m_{2} m_{3} m_{4} m_{5}$ such that $m_{i}$ is an element in the $i$ th row of the next list

$$
\begin{aligned}
& 1, x_{0}, \\
& 1, x_{1}, x_{1} x_{0}, \\
& 1, x_{\omega} x_{0} x_{1}, \\
& 1, x_{\omega}, x_{\omega} x_{0}, \\
& 1, x_{\omega^{2}} .
\end{aligned}
$$

By (5) the weight of a monomial $x_{i_{1}} \cdots x_{i_{\ell}} \in T(V)$ is $g_{i_{1}}^{-1} \cdots g_{i_{\ell}}^{-1}$. Set $g_{\text {top }}=g_{0}^{-1} g_{1}^{-1} g_{0}^{-1} g_{\omega}^{-1} g_{0}^{-1} g_{1}^{-1} g_{\omega}^{-1} g_{0}^{-1} g_{\omega^{2}}^{-1}$. An integral of $\mathcal{B}(V)$ is

$$
m_{\text {top }}=x_{0} x_{1} x_{0} x_{\omega} x_{0} x_{1} x_{\omega} x_{0} x_{\omega^{2}} \in \mathbb{B}\left[g_{t o p}\right] .
$$


Lemma 10. Let $G$ be a finite group with a faithful principal YD-realization $\left(\cdot, g, \chi_{G}\right)$ of $\left(\operatorname{Aff}\left(\mathbb{F}_{4}, \omega\right),-1\right)$. Hence

(a) $\operatorname{Supp} \mathcal{B}(V)=\operatorname{Supp} \mathbb{B} \subset G^{\prime}$.

(b) $G^{\prime} \longmapsto \mathbb{F}_{4} \rtimes_{\omega} C_{6}, g_{i} \mapsto(i, t)$ is an epimorphism of groups.

(c) If $z \in T(V)[e]$, then $\mathbb{B}[e]=\left\{1, b_{1}, b_{2}, b_{3}, b_{4}, b_{5}\right\}$ where

$$
\begin{array}{ll}
b_{1}=x_{0} x_{1} x_{0} x_{\omega} x_{0} x_{\omega^{2}}, & b_{2}=x_{0} x_{\omega} x_{0} x_{1} x_{\omega} x_{\omega^{2}}, \quad b_{3}=x_{1} x_{0} x_{\omega} x_{0} x_{1} x_{\omega^{2}} \\
b_{4}=x_{1} x_{\omega} x_{0} x_{1} x_{\omega} x_{0}, & b_{5}=x_{0} x_{1} x_{\omega} x_{0} x_{1} x_{\omega} .
\end{array}
$$

(d) Let $y=\sum_{i \in \mathbb{F}_{4}} x_{i}$ and $U=\mathbb{k}\left\{x_{0}-x_{1}, x_{0}-x_{\omega}, x_{0}-x_{\omega^{2}}\right\}$. Then $\mathbb{k} y$ and $U$ are simple $\mathbb{k}^{G}$-comodules such that $V=\mathbb{k} y \oplus U$.

Proof (a) holds since the elements of $\mathbb{B}$ are $\mathbb{k}^{G}$-homogeneous and $\mathcal{B}(V)$ is a $\mathbb{k}^{G}$-module algebra.

(b) By $[\mathrm{AG} 2$, Lemma $1.9(1)]$, the quotient of $G^{\prime}$ by its center $\mathcal{Z}\left(G^{\prime}\right)$ is isomorphic to $\operatorname{Inn}_{\triangleright} \operatorname{Aff}\left(\mathbb{F}_{4}, \omega\right)=\mathbb{F}_{4} \rtimes_{\omega} C_{3}$ via $\overline{g_{i}} \mapsto(i, t), i \in \mathbb{F}_{4}$. Then $G^{\prime} /\left(\mathcal{Z}\left(G^{\prime}\right) \cap \operatorname{ker} \chi_{G}\right) \simeq \mathbb{F}_{4} \rtimes_{\omega} C_{3} \times C_{2} \simeq \mathbb{F}_{4} \rtimes_{\omega} C_{6}$.

(c) If $z \in \mathbb{B}[e]$, then $\left\{1, b_{1}, b_{2}, b_{3}, b_{4}, b_{5}\right\} \subseteq \mathbb{B}[e]$ since $g_{i} g_{j}=g_{i \triangleright j} g_{i}$. The other inclusion follows using (b).

(d) is equivalent to prove that $\mathbb{k} y$ and $U$ are simple $\mathbb{k} G$-modules via the action $g \cdot x_{i}=\chi_{G}(g) x_{g \cdot i}, i \in \mathbb{F}_{4}$. Clearly, $\mathbb{k} y$ and $U$ are $\mathbb{k} G$-submodules and $\mathbb{k} y$ is $\mathbb{k} G$-simple. Moreover, it is an straightforward computation to show that $U$ is $\mathbb{k} G^{\prime}$-simple and therefore $\mathbb{k} G$-simple.

\subsection{Copointed Hopf algebras over $\operatorname{Aff}\left(\mathbb{F}_{4}, \omega\right)$}

The copointed Hopf algebras over $\mathbb{k}^{G}$ whose infinitesimal braiding arises from a principal YD-realization of the affine rack Aff $\left(\mathbb{F}_{4}, \omega\right)$ with the constant 2-cocycle -1 are classified in [GIV] as follows.

By (5) the smash product Hopf algebra $T(V) \# \mathbb{k}^{G}$ is defined by

$$
\begin{aligned}
\delta_{t} x_{i} & =x_{i} \delta_{g_{i} t} \quad \text { and } \\
\Delta\left(x_{i}\right) & =x_{i} \otimes 1+\sum_{t \in G} \chi_{G}(t) \delta_{t^{-1}} \otimes x_{t \cdot i} \quad \forall t \in G, i \in X .
\end{aligned}
$$

Definition 1 Let $\lambda \in \mathbb{k}$ and assume $z \in T(V)[e]$. The Hopf algebra $\mathcal{A}_{G, \lambda}$ is the quotient of $T(V) \# \mathbb{k}^{G}$ by the ideal generated by (6) and $z-f$ where

$$
f=\lambda\left(1-\chi_{z}^{-1}\right) \quad \text { and } \quad \chi_{z}=\chi_{G}^{6} .
$$

Notice that if either $\lambda=0$ or $\chi_{z}=1$, then $\mathcal{A}_{G, \lambda}=\mathcal{B}(V) \# \mathbb{k}^{G}$.

The next theorem is [GIV, Main theorem 2 and Theorem 4.5].

Theorem 11. Let $H$ be a copointed Hopf algebra over $\mathbb{k}^{G}$ whose infinitesimal braiding arises from a principal $Y D$-realization of the affine rack $\operatorname{Aff}\left(\mathbb{F}_{4}, \omega\right)$ with the constant 2-cocycle -1 . 
(a) If $G=G^{\prime}$, then $H \simeq \mathcal{B}(V) \# \mathbb{k}^{G}$.

(b) If $z \in T(V)^{\times}$, then $H \simeq \mathcal{B}(V) \# \mathbb{k}^{G}$.

(c) If $z \in T(V)[e]$, then $H \simeq \mathcal{A}_{G, \lambda}$ for some $\lambda \in \mathbb{k}$.

(d) $\mathcal{A}_{G, \lambda}$ is a cocycle deformation of $\mathcal{A}_{G, \lambda^{\prime}}$, for all $\lambda, \lambda^{\prime} \in \mathbb{k}$.

(e) $\mathcal{A}_{G, \lambda}$ is a lifting of $\mathcal{B}(V)$ over $\mathbb{k}^{G}$ for all $\lambda, \lambda^{\prime} \in \mathbb{k}$.

(f) $\mathcal{A}_{G, \lambda} \simeq \mathcal{A}_{G, 1} \nsucceq \mathcal{A}_{G, 0}$ for all $\lambda \in \mathbb{R}^{*}$.

We think of $\mathcal{A}_{G, \lambda}$ as an algebra presented by generators $\left\{x_{i}, \delta_{g}: i \in \mathbb{F}_{4}, g \in\right.$ $G\}$ and relations:

$$
\begin{gathered}
\delta_{g} x_{i}=x_{i} \delta_{g_{i} g}, \quad x_{i}^{2}=0, \quad \delta_{g} \delta_{h}=\delta_{g}(h) \delta_{g}, \quad 1=\sum_{g \in G} \delta_{g}, \\
x_{0} x_{\omega}+x_{\omega} x_{1}+x_{1} x_{0}=0=x_{0} x_{\omega^{2}}+x_{\omega^{2}} x_{\omega}+x_{\omega} x_{0}, \\
x_{1} x_{\omega^{2}}+x_{0} x_{1}+x_{\omega^{2}} x_{0}=0=x_{\omega} x_{\omega^{2}}+x_{1} x_{\omega}+x_{\omega^{2}} x_{1} \quad \text { and } \\
x_{\omega} x_{0} x_{1} x_{\omega} x_{0} x_{1}+x_{1} x_{\omega} x_{0} x_{1} x_{\omega} x_{0}+x_{0} x_{1} x_{\omega} x_{0} x_{1} x_{\omega}=f,
\end{gathered}
$$

for all $i \in \mathbb{F}_{4}$ and $g \in G$. Since $\chi_{z}\left(g_{i}\right)=1$, it holds that

$$
f x_{i}=x_{i} f \quad \forall i \in \mathbb{F}_{4} .
$$

A basis for $\mathcal{A}_{G, \lambda}$ is $\mathbb{A}=\left\{x \delta_{g} \mid x \in \mathbb{B}, g \in G\right\}$ and a basis for the Verma module $M_{g}$ is $\mathbb{M}=\left\{x_{i_{1}} \cdots x_{i_{s}} \delta_{g} \in \mathbb{B} \delta_{g}\right\}$.

Proposition 12. $\mathcal{A}_{G, \lambda}$ is not quasitriangular.

Proof Let $A$ be a pointed Hopf subalgebra of $\mathcal{A}_{G, \lambda}$ with abelian group of group-like elements. Then $A$ is generated by skew-primitives and group-likes elements by [An, Theorem 2].

Let $y=\sum_{i \in \mathbb{F}_{4}} x_{i}$. The space of skew-primitives of $\mathcal{A}_{G, \lambda}$ is $\mathbb{k} G\left(\mathcal{A}_{G, \lambda}\right) \oplus$ $\mathbb{k} y \mathbb{k} G\left(\mathcal{A}_{G, \lambda}\right)$ by Lemma 10 (d). Then $A$ is generated by $y$ and $G(A)$. By $(9)$, $y^{2}=0$ and hence $A \subseteq\left(\mathbb{k}[y] /\left\langle y^{2}\right\rangle\right) \# \mathbb{k} G(A)$. Therefore $\mathcal{A}_{G, \lambda}$ is not quasitriangular by Lemma 8 .

\section{Representation theory of $\mathcal{A}_{G, \lambda}$}

Let $\left(\cdot, g, \chi_{G}\right)$ be a faithful principal YD-realization of $\left(\operatorname{Aff}\left(\mathbb{F}_{4}, \omega\right),-1\right)$ over a fixed finite group $G$. Let $V \in \mathbb{k}_{\mathbb{k}^{G}}^{\mathbb{k}^{G}} \mathcal{Y D}$ be as in (5).

Also we fix $\lambda \in \mathbb{R}^{*}$ and assume $z \in T(V)[e]$ and $\chi_{z} \neq 1$. In this section we study the Hopf algebra $\mathcal{A}_{G, \lambda}$, Definition 1.

For $g \in G \backslash \operatorname{ker} \chi_{z}$, we define

$$
\begin{array}{llrl}
\mathbf{e}_{1}^{g}=-\frac{1}{f(g)} b_{1} \delta_{g}, & \mathbf{e}_{2}^{g}=-\frac{1}{f(g)} b_{2} \delta_{g}, & \mathbf{e}_{3}^{g}=\frac{1}{f(g)} b_{3} \delta_{g}, \\
\mathbf{e}_{4}^{g}=\frac{1}{f(g)}\left(b_{4}-b_{3}\right) \delta_{g}, & \mathbf{e}_{5}^{g}=\frac{1}{f(g)}\left(b_{5}+b_{1}\right) \delta_{g} & \text { and }
\end{array}
$$




$$
\mathbf{e}_{6}^{g}=\delta_{g}+\frac{1}{f(g)}\left(b_{2}-b_{4}-b_{5}\right) \delta_{g}
$$

where $b_{1}, b_{2}, b_{3}, b_{4}, b_{5} \in \mathcal{A}_{G, \lambda}$ are as in Lemma 10 (c).

Lemma 13. A complete set of orthogonal primitive idempotents of $\mathcal{A}_{G, \lambda}$ is

$$
\mathcal{E}:=\left\{\delta_{h}, \mathbf{e}_{1}^{g}, \mathbf{e}_{2}^{g}, \mathbf{e}_{3}^{g}, \mathbf{e}_{4}^{g}, \mathbf{e}_{5}^{g}, \mathbf{e}_{6}^{g} \mid h \in \operatorname{ker} \chi_{z}, g \in G \backslash \operatorname{ker} \chi_{z}\right\} .
$$

Proof By Lemma $10(\mathrm{c}),\left\{b_{i} \delta_{g} \mid 1 \leq i \leq 6\right\}$ is a basis of $\mathcal{B}(V)[e] \delta_{g}$ for all $g \in G$. By (9) and (10), it holds that:

$$
\begin{aligned}
& b_{1}^{2}=-b_{1} f, \quad b_{1} b_{2}=0, \quad b_{1} b_{3}=0, \quad b_{1} b_{4}=0, \quad b_{1} b_{5}=b_{1} f, \\
& b_{2} b_{1}=0, \quad b_{2}^{2}=-b_{2} f, \quad b_{2} b_{3}=0, \quad b_{2} b_{4}=0, \quad b_{2} b_{5}=0, \\
& b_{3} b_{1}=0, \quad b_{3} b_{2}=0, \quad b_{3}^{2}=b_{3} f, \quad b_{3} b_{4}=b_{3} f, \quad b_{3} b_{5}=0, \\
& b_{4} b_{1}=0, \quad b_{4} b_{2}=0, \quad b_{4} b_{3}=b_{3} f, \quad b_{4}^{2}=b_{4} f, \quad b_{4} b_{5}=0 \text {, } \\
& b_{5} b_{1}=b_{1} f, \quad b_{5} b_{2}=0, \quad b_{5} b_{3}=0, \quad b_{5} b_{4}=0, \quad b_{5}^{2}=b_{5} f
\end{aligned}
$$

Therefore $\mathcal{E}_{h}=\left\{\delta_{h}\right\}$ is a complete set of orthogonal primitive $h$-idempotent for all $h \in \operatorname{ker} \chi_{z}$. If $g \in G \backslash \operatorname{ker} \chi_{z}$, we apply Lemma 7 to the ordered set

$$
\left\{-\frac{1}{f(g)} b_{1} \delta_{g},-\frac{1}{f(g)} b_{2} \delta_{g}, \frac{1}{f(g)} b_{3} \delta_{g}, \frac{1}{f(g)} b_{4} \delta_{g}, \frac{1}{f(g)} b_{5} \delta_{g}, \delta_{g}\right\}
$$

and hence $\mathcal{E}_{g}=\left\{\mathbf{e}_{i}^{g} \mid 1 \leq i \leq 6\right\}$ is a complete set of orthogonal primitive $g$-idempotent. Then $\mathcal{E}=\cup_{g \in G} \mathcal{E}_{g}$.

Let $M$ be an $\mathcal{A}_{G, \lambda}$-module. Since $\mathcal{A}_{G, \lambda}$ is a quotient of $T(V) \# \mathbb{k}^{G}, M$ also is a $T(V) \# \mathbb{k}^{G}$-module. Moreover, $M$ is a $T(V) \# \mathbb{k}^{\text {ker } \chi_{z} \text {-module if Supp } M \subseteq}$ ker $\chi_{z}$ since $T(V) \# \mathbb{k}^{\operatorname{ker} \chi_{z}}$ is a subalgebra of $T(V) \# \mathbb{k}^{G}$, cf. Example $9(3)$.

Lemma 14. Let $h \in \operatorname{ker} \chi_{z}$.

(a) If $M$ is an $\mathcal{A}_{G, \lambda}$-module with $\operatorname{Supp} M \subseteq \operatorname{ker} \chi_{z}$, then $M$ is a module over $\mathcal{B}(V) \# \mathbb{k}^{\operatorname{ker} \chi_{z}}$

(b) $M_{h}$ is a free $\mathcal{B}(V)$-module of rank 1 generated by $\delta_{h}$.

(c) $\chi_{h}: \mathcal{A}_{G, \lambda} \rightarrow \mathbb{k}$ is an algebra map.

(d) $\operatorname{top}\left(M_{h}\right) \simeq \mathbb{k}_{h}$ and $\operatorname{soc}\left(M_{h}\right) \simeq \mathbb{k}_{g_{\text {top }} h}$.

(e) $\int_{\mathcal{A}_{G, \lambda}}^{l}=\operatorname{soc}\left(M_{g_{\text {top }}^{-1}}\right)$ and $\chi_{g_{\text {top }}}$ is the distinguished group-like element.

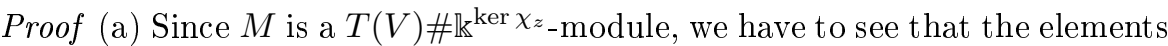
in (6) and $z$ act by zero over $M$. This is true for the first elements because they are zero in $\mathcal{A}_{G, \lambda}$. If $h \in \operatorname{ker} \chi_{z}$, then $f \delta_{h}=0$ and hence $z \cdot M[h]=f \cdot\left(\delta_{h} \cdot M\right)=0$. (b) follows from (a). (c) is clear. (d) and (e) follows from (b) and Lemma 6.

For each $\mathbf{e}_{i}^{g} \in \mathcal{E}$, we set $L_{i}^{g}=\mathcal{A}_{G, \lambda} \mathbf{e}_{i}^{g}$.

Lemma 15. (a) $L_{i}^{g}$ is an injective and projective simple module of dimension 12 for all $\mathbf{e}_{i}^{g} \in \mathcal{E}$. 
(b) There exist $\mathbb{k}^{G}$-submodules $L_{1}, \ldots, L_{6} \subset \mathcal{B}(V)$ such that $\mathcal{B}(V)=L_{1} \oplus \cdots \oplus$ $L_{6}$ and $L_{i}^{g}=L_{i} \delta_{g}$ for all $i=1, \ldots, 6$ and $g \in G$.

(c) $\operatorname{Supp} L_{i} \neq \operatorname{Supp} L_{j}$ and $\operatorname{Supp} L_{i}^{g}=\left(\operatorname{Supp} L_{i}\right) g$ for all $1 \leq i, j \leq 6$ and $g \in G$.

(d) $L_{i}^{g} \simeq L_{j}^{h}$ if and only if $\left(\operatorname{Supp} L_{i}\right) g=\left(\operatorname{Supp} L_{j}\right) h$.

Proof (a) Let $v=\overline{\mathbf{e}_{i}^{g}} \in \operatorname{top}\left(L_{i}^{g}\right)$. Since $f(g) v=z \cdot v=\left(x_{\omega} x_{0} x_{1}\right)^{2} \cdot v+b_{4} \cdot v+$ $b_{5} \cdot v \neq 0$, there are $x_{i_{6}}, \ldots, x_{i_{1}} \in \mathcal{A}_{G, \lambda}$ such that $x_{i_{\ell}} \cdots x_{i_{1}} \cdot v \neq 0$ for all $\ell=1, \ldots, 6$.

We claim that $\operatorname{dim} \operatorname{top}\left(L_{i}^{g}\right) \geq 11$. In fact, if $1 \leq \ell<6$, then by (6)

$$
\begin{aligned}
& x_{i_{\ell+1}} x_{i_{\ell}} \cdots x_{i_{1}} \cdot v= \\
& \quad-x_{i_{\ell}} x_{(\omega+1) i_{\ell}+\omega i_{\ell+1}} \cdots x_{i_{1}} \cdot v-x_{(\omega+1) i_{\ell}+\omega i_{\ell+1}} x_{i_{\ell+1}} \cdots x_{i_{1}} \cdot v \neq 0
\end{aligned}
$$

and hence $x_{(\omega+1) i_{\ell}+\omega i_{\ell+1}} \cdots x_{i_{1}} \cdot v \neq 0$ or $x_{i_{\ell+1}} \cdots x_{i_{1}} \cdot v \neq 0$. Therefore using Lemma $10(\mathrm{~b})$, we see that \# $\operatorname{Supp} \operatorname{top}\left(L_{i}^{g}\right) \geq 11$.

Now, we show that $L_{i}^{g}=\operatorname{soc}\left(L_{i}^{g}\right)=\operatorname{top}\left(L_{i}^{g}\right)$ and (a) follows. Otherwise, $\operatorname{dim} L_{i}^{g} \geq 22$ since $\operatorname{dim} \operatorname{top}\left(L_{i}^{g}\right)=\operatorname{dim} \operatorname{soc}\left(L_{i}^{g}\right)$ by [CR, Lemma 58.4]. But the above claim holds for all $i$ and hence $72=\operatorname{dim} M_{g} \geq 22+5 \cdot 11$, a contradiction.

(b) follows from Tables 1-6 in Appendix. (c) $\operatorname{Supp} L_{i}^{g}=\left(\operatorname{Supp} L_{i}\right) g$ follows from (b). If $G^{\prime}=\mathbb{F}_{4} \rtimes C_{6}$, then $\operatorname{Supp} L_{i} \neq \operatorname{Supp} L_{j}$ by Table 7 in Appendix and therefore for any $G^{\prime}$ by Lemma 10 (b). (d) follows from (c) and Lemma 3.

We consider the product set $\{1,2,3,4,5,6\} \times G$ with the equivalence relation $i \times g \sim j \times h$ if and only if $\left(\operatorname{Supp} L_{i}\right) g=\left(\operatorname{Supp} L_{j}\right) h$. Let $\mathfrak{X}$ be the set of equivalence classes of $\sim$. We denote by $[i, g]$ the equivalence class of $i \times g$. By Lemma $15(\mathrm{~d})$, we can define $L_{[i, g]}=L_{i}^{g}$.

Theorem 16. Every simple $\mathcal{A}_{G, \lambda}$-module is isomorphic to either

$$
\begin{aligned}
& \mathbb{k}_{g} \quad \text { for a unique } g \in \operatorname{ker} \chi_{z} \text { or } \\
& L_{[i, g]} \text { for a unique }[i, g] \in \mathfrak{X} \text {. }
\end{aligned}
$$

In particular, (up to isomorphism) there are $\left|\operatorname{ker} \chi_{z}\right|$ one-dimensional simple $\mathcal{A}_{G, \lambda}$-modules and $\frac{\left(|G|-\left|\operatorname{ker} \chi_{z}\right|\right)}{2}$ 12-dimensional simple $\mathcal{A}_{G, \lambda}$-modules.

Proof It follows from Lemmata 13, 14 and 15.

Example 2 Assume $G^{\prime}=\mathbb{F}_{4} \rtimes C_{6}$ and let $g \in G \backslash$ ker $\chi_{z}$. The set $\mathfrak{X}$ is completely defined by the equivalence class $[1, g]$ which is

$$
\begin{gathered}
\left\{1 \times g, 2 \times\left(1, t^{2}\right) g,(3, t g), 4 \times\left(\omega, t^{2}\right) g, 5 \times(1, t) g, 6 \times(\omega, 1) g, 1 \times\left(0, t^{3}\right) g\right. \\
\left.2 \times\left(1, t^{5}\right) g, 3 \times\left(0, t^{4}\right) g, 4 \times\left(\omega, t^{5}\right) g, 5 \times\left(1, t^{4}\right) g, 6 \times\left(\omega, t^{3}\right) g\right\} .
\end{gathered}
$$

Hence

$$
L_{[1, g]}=L_{1}^{g} \simeq L_{2}^{\left(1, t^{2}\right) g} \simeq L_{3}^{(0, t) g} \simeq L_{4}^{\left(\omega, t^{2}\right) g} \simeq L_{5}^{(1, t) g} \simeq L_{6}^{(\omega, 1) g} \simeq
$$




$$
L_{1}^{\left(0, t^{3}\right) g} \simeq L_{2}^{\left(1, t^{5}\right) g} \simeq L_{3}^{\left(0, t^{4}\right) g} \simeq L_{4}^{\left(\omega, t^{5}\right) g} \simeq L_{5}^{\left(1, t^{4}\right) g} \simeq L_{6}^{\left(\omega, t^{3}\right) g} .
$$

Note that $i \times g \sim i \times\left(0, t^{3}\right) g$ for all $i$, then $L_{i}^{g} \simeq L_{i}^{\left(0, t^{3}\right) g}$.

In fact, $\left(\operatorname{Supp} L_{2}\right)\left(1, t^{2}\right)=\operatorname{Supp} L_{1}$, see Tables 1 and 2 . Then $L_{1}^{g} \simeq L_{2}^{\left(1, t^{2}\right) g}$ by Lemma 15 (d). The other isomorphisms are obtained in the same way.

4.1 Decomposition of the category of $\mathcal{A}_{G, \lambda}$-modules

Fix $\lambda \in \mathbb{R}^{*}$ and assume $z \in T(V)[e]$ and $\chi_{z} \neq 1$. Let $I \subset\{1,2,3,4,5,6\} \times G$ be a set of representative of the equivalence classes of $\sim$. Let $M$ be an $\mathcal{A}_{G, \lambda^{-}}$ module.

If $i \times g \in I$, then $d_{[i, g]}^{M}=\operatorname{dim}\left(\mathbf{e}_{i}^{g} \cdot M\right)$ is the number of composition factors of $M$ which are isomorphic to $L_{[i, g]}$ [CR, Theorem 54.16]. The number $d_{[i, g]}^{M}$ can be calculated keeping in mind Lemma 1 (d). Since $L_{[i, g]}$ is projective and injective by Lemma 15, there is a submodule $N \subseteq M$ such that $\operatorname{Supp} N \subseteq$ ker $\chi_{z}$ and

$$
M=N \oplus \bigoplus_{j \in I}\left(L_{j}\right)^{d_{[i, g]}^{M}} .
$$

Moreover, $N$ is a $\mathcal{B}(V) \# \mathbb{k}^{\text {ker } \chi_{z}}$-module by Lemma 14 (a).

4.2 Representation type of $\mathcal{A}_{G, \lambda}$

Now, we do not make any assumptions on $z$ and $\lambda$ can be zero. Let $\mathbb{k}_{g}$ and $\mathbb{k}_{h}$ be one-dimensional $\mathcal{A}_{G, \lambda}$-modules such that $g=g_{i}^{-1} h \in \operatorname{ker} \chi_{z}$ for some $i \in \mathbb{F}_{4}$. We define the $\mathcal{A}_{G, \lambda}$-module $M_{g, h}=\mathbb{k}\left\{w_{h}, w_{g}\right\}$ by $\mathbb{k} w_{g} \simeq \mathbb{k}_{g}$ as $\mathcal{A}_{G, \lambda}$-modules, $w_{h} \in M[h]$ and $x_{j} w_{h}=\delta_{j, i} w_{g}$ for all $j \in \mathbb{F}_{4}$.

Proposition 17. The extensions of one-dimensional $\mathcal{A}_{G, \lambda}$-modules are either trivial or isomorphic to $M_{g, h}$ for some $g, h \in \operatorname{ker} \chi_{z}$. Hence $\mathcal{A}_{G, \lambda}$ is of wild representation type.

Proof Let $M$ be an extension of $\mathbb{k}_{h}$ by $\mathbb{k}_{g}$. Then $M=M[g] \oplus M[h]$ as $\mathbb{k}^{G}$ modules and $M[g] \simeq \mathbb{k}_{g}$ as $\mathcal{A}_{G, \lambda}$-modules. Since $x_{i} \cdot M[h] \subset M\left[g_{i}^{-1} h\right]$, the first part follows.

For the second part we can easily see that $\operatorname{Ext}_{\mathcal{A}_{G, \lambda}}^{1}\left(\mathbb{k}_{g}, \mathbb{k}_{h}\right)$ is either 1 or 0 for all $g, h \in \operatorname{ker} \chi_{z}$. Then the separated quiver of $\mathcal{A}_{G, \lambda}$ is wild. The details for this proof are similar to [AV2, Proposition 26].

4.3 Is $\mathcal{A}_{G, \lambda}$ spherical?

A Hopf algebra $H$ is spherical [BaW1] if there is $\omega \in G(H)$ such that

$$
\begin{aligned}
\mathcal{S}^{2}(x) & =\omega x \omega^{-1} \quad \forall x \in H \text { and } \\
\operatorname{tr}_{V}(\omega) & =\operatorname{tr}_{V}\left(\omega^{-1}\right) \quad \forall V \in \operatorname{Irr} H \quad \text { by [AAGTV, Proposition 2.1]. }
\end{aligned}
$$


Proposition 18. $\mathcal{B}(V) \# \mathbb{K}^{G}$ is spherical iff $\chi_{G}^{2}=1$. Moreover, $\left(\mathcal{A}_{G, \lambda}, \chi_{G}\right)$ with $\lambda \neq 0$ is spherical iff $\left(\chi_{G \mid \operatorname{ker} \chi_{z}}\right)^{2}=1$.

Proof It is a straightforward computation to see that $\chi_{G}$ satisfies (12) using (8). Let $V \in \operatorname{Irr} \mathcal{A}_{G, \lambda}$. If $\operatorname{dim} V=12$, then $V$ is projective and therefore $\operatorname{tr}_{V}\left(\chi_{G}^{ \pm 1}\right)=0$ [BaW2, Proposition 6.10]. If $V=\mathbb{k}_{h}$ with $h \in \operatorname{ker} \chi_{z}$, then (13) holds iff $\chi_{G}(h)= \pm 1$.

Example 3 Let $\left(\cdot, g, \chi_{G}\right)$ be the faithful principal YD-realization in Example 9 (2). Then $\left(\mathcal{A}_{G, \lambda}, \chi_{G}\right)$ is a spherical Hopf algebra with non involutory pivot.

Any spherical Hopf algebra $H$ has an associated tensor category $\operatorname{Rep}(H)$ which is a quotient of $\operatorname{Rep}(H)$, see [AAGMV, BaW1, BaW2] for the background of this subject. Moreover, $\operatorname{Rep}(H)$ is semisimple but rarely is a fusion category in the sense of [ENO], $i$. e. $\underline{\operatorname{Rep}}(H)$ rarely has a finite number of irreducibles. One hopes to find new examples of fusion categories as tensor subcategories of $\operatorname{Rep}(H)$ for a suitable $H$. However, this is not possible for $H=\mathcal{A}_{G, \lambda}$, see below.

Remark 19. Assume that $\left(\mathcal{A}_{G, \lambda}, \chi_{G}\right)$ is spherical. Then only the one-dimensional simple modules survive in $\operatorname{Rep}\left(\mathcal{A}_{G, \lambda}\right)$ since the other simple modules are projective. Then $\operatorname{Rep}\left(\mathcal{A}_{G, \lambda}\right)$ is equivalent to $\operatorname{Rep}\left(\mathcal{B}(V) \# \mathbb{k}^{\operatorname{ker} \chi_{z}}\right)$ by Subsection 4.1 , where the pivot $\chi_{G \mid \operatorname{ker} \chi_{z}}$ is involutory. Hence any fusion subcategory of $\operatorname{Rep}\left(\mathcal{A}_{G, \lambda}\right)$ is equivalent to $\operatorname{Rep}(K)$, with $K$ a semisimple quasi-Hopf algebra, by [AAGTV, Proposition 2.12].

\section{Appendix}

The next tables describe the structure of the 12-dimensional simple modules of $\mathcal{A}_{G, \lambda}$. These were used in Lemma 15 .

Table 1 Action of the generators $x_{i}$ on $L_{1}^{g}=\mathcal{A}_{G, \lambda} \mathbf{e}_{1}^{g}$

\begin{tabular}{l|c|c|c|c}
\hline \hline Linear basis of $L_{1}^{g}$ & $x_{0} \cdot$ & $x_{1} \cdot$ & $x_{\omega} \cdot$ & $x_{\omega^{2}} \cdot$ \\
\hline$c_{1}=x_{0} x_{1} x_{0} x_{\omega} x_{0} x_{1} x_{\omega} x_{0} x_{\omega^{2}} \delta_{g}$ & 0 & 0 & $-f(g) c_{6}$ & $-f(g) c_{10}$ \\
$c_{2}=x_{0} x_{1} x_{0} x_{\omega} x_{0} x_{\omega^{2}} \delta_{g}=-f(g) \mathbf{e}_{1}^{g}$ & 0 & 0 & $-c_{5}$ & $-c_{9}$ \\
$c_{3}=x_{0} x_{1} x_{\omega} x_{0} x_{1} x_{\omega} x_{0} x_{\omega^{2}} \delta_{g}$ & 0 & $c_{1}$ & $f(g) c_{12}$ & 0 \\
$c_{4}=x_{0} x_{1} x_{\omega} x_{0} x_{\omega^{2}} \delta_{g}$ & 0 & $c_{2}$ & $c_{11}$ & 0 \\
$c_{5}=x_{0} x_{\omega} x_{0} x_{1} x_{\omega} x_{0} x_{\omega^{2}} \delta_{g}$ & 0 & $c_{7}$ & 0 & $-c_{3}$ \\
$c_{6}=x_{0} x_{\omega} x_{0} x_{\omega^{2}} \delta_{g}$ & $c_{1}$ & 0 & 0 & $-c_{4}$ \\
$c_{7}=x_{1} x_{0} x_{\omega} x_{0} x_{1} x_{\omega} x_{0} x_{\omega^{2}} \delta_{g}$ & $c_{2}$ & 0 & 0 & $-f(g) c_{12}$ \\
$c_{8}=x_{1} x_{0} x_{\omega} x_{0} x_{\omega^{2}} \delta_{g}$ & $c_{3}$ & 0 & $-c_{7}$ & 0 \\
$c_{9}=x_{1} x_{\omega} x_{0} x_{1} x_{\omega} x_{0} x_{\omega^{2}} \delta_{g}$ & $c_{4}$ & 0 & $-c_{8}$ & 0 \\
$c_{10}=x_{1} x_{\omega} x_{0} x_{\omega^{2}} \delta_{g}$ & $c_{5}$ & $c_{9}$ & 0 & 0 \\
$c_{11}=x_{\omega} x_{0} x_{1} x_{\omega} x_{0} x_{\omega^{2}} \delta_{g}$ & $c_{6}$ & $c_{10}$ & 0 & 0 \\
$c_{12}=x_{\omega} x_{0} x_{\omega^{2}} \delta_{g}$ & \multicolumn{4}{|c}{} \\
\hline
\end{tabular}


Table 2 Action of the generators $x_{i}$ on $L_{2}^{g}=\mathcal{A}_{G, \lambda} \mathbf{e}_{2}^{g}$

\begin{tabular}{l|c|c|c|c}
\hline \hline Linear basis of $L_{2}^{g}$ & $x_{0} \cdot$ & $x_{1} \cdot$ & $x_{\omega} \cdot$ & $x_{\omega^{2}} \cdot$ \\
\hline$c_{1}=x_{0} x_{1} x_{0} x_{\omega} x_{0} x_{1} x_{\omega} x_{\omega^{2}} \delta_{g}$ & 0 & 0 & $c_{6}$ & $-f(g) c_{10}$ \\
$c_{2}=x_{0} x_{1} x_{0} x_{\omega} x_{\omega^{2}} \delta_{g}$ & 0 & 0 & $-c_{5}$ & $-c_{9}$ \\
$c_{3}=x_{0} x_{1} x_{\omega} x_{0} x_{1} x_{\omega} x_{\omega^{2}} \delta_{g}$ & 0 & $c_{1}$ & $-c_{12}$ & 0 \\
$c_{4}=x_{0} x_{1} x_{\omega} x_{\omega^{2}} \delta_{g}$ & 0 & $c_{2}$ & $c_{11}$ & 0 \\
$c_{5}=x_{0} x_{\omega} x_{0} x_{1} x_{\omega} x_{\omega^{2}} \delta_{g}=f(g) \mathbf{e}_{2}^{g}$ & 0 & $c_{7}$ & 0 & $-c_{3}$ \\
$c_{6}=x_{0} x_{1} x_{0} x_{\omega} x_{0} x_{1} x_{\omega} x_{0} x_{\omega^{2}} \delta_{g}$ & & $-f(g) c_{8}$ & 0 & $f(g) c_{4}$ \\
$\quad-x_{0} x_{\omega} x_{\omega^{2}} \delta_{g}$ & $c_{1}$ & 0 & & \\
$c_{7}=x_{1} x_{0} x_{\omega} x_{0} x_{1} x_{\omega} x_{\omega^{2}} \delta_{g}$ & $c_{3}$ & 0 & 0 & $-c_{12}$ \\
$c_{8}=x_{1} x_{0} x_{\omega} x_{\omega} \delta_{g}$ & $c_{4}$ & 0 & $-c_{7}$ & 0 \\
$c_{9}=x_{1} x_{\omega} x_{0} x_{1} x_{\omega} x_{\omega^{2}} \delta_{g}$ & $c_{5}$ & $c_{9}$ & 0 & 0 \\
$c_{10}=x_{1} x_{\omega} x_{\omega^{2}} \delta_{g}$ & $-f(g) c_{10}$ & 0 & 0 \\
$c_{11}=x_{\omega} x_{0} x_{1} x_{\omega} x_{\omega^{2}} \delta_{g}$ & & \\
$c_{12}=x_{1} x_{0} x_{\omega} x_{0} x_{1} x_{\omega} x_{0} x_{\omega^{2}} \delta_{g}-x_{\omega} x_{\omega^{2}} \delta_{g}$ & $c_{6}$ & $-f$ & 0 \\
\hline
\end{tabular}

Table 3 Action of the generators $x_{i}$ on $L_{3}^{g}=\mathcal{A}_{G, \lambda} \mathbf{e}_{3}^{g}$

\begin{tabular}{l|c|c|c|c}
\hline \hline Linear basis of $L_{3}^{g}$ & $x_{0} \cdot$ & $x_{1} \cdot$ & $x_{\omega} \cdot$ & $x_{\omega^{2}}$ \\
\hline$c_{1}=x_{0} x_{1} x_{0} x_{\omega} x_{0} x_{1} x_{\omega^{2}} \delta_{g}$ & 0 & 0 & $c_{6}$ & $-c_{10}$ \\
$c_{2}=x_{0} x_{1} x_{0} x_{\omega^{2}} \delta_{g}$ & 0 & 0 & $-c_{5}$ & $-c_{9}$ \\
$c_{3}=x_{0} x_{1} x_{\omega} x_{0} x_{1} x_{\omega^{2}} \delta_{g}$ & 0 & $c_{1}$ & $c_{12}$ & 0 \\
$c_{4}=x_{0} x_{1} x_{\omega^{2}} \delta_{g}$ & 0 & $c_{2}$ & $c_{11}$ & 0 \\
$c_{5}=x_{0} x_{\omega} x_{0} x_{1} x_{\omega^{2}} \delta_{g}$ & 0 & $c_{7}$ & 0 & $-c_{3}$ \\
$c_{6}=x_{0} x_{1} x_{\omega} x_{0} x_{1} x_{\omega} x_{0} x_{\omega^{2}} \delta_{g}$ & $c_{8}$ & 0 & $f(g) c_{4}$ \\
$\quad-f(g) x_{0} x_{\omega^{2}} \delta_{g}$ & $-f(g) c_{2}$ & 0 & 0 & $-f(g) c_{11}$ \\
$c_{7}=x_{1} x_{0} x_{\omega} x_{0} x_{1} x_{\omega^{2}} \delta_{g}=f(g) \mathbf{e}_{3}^{g}$ & & & & \\
$c_{8}=x_{0} x_{1} x_{0} x_{\omega} x_{0} x_{1} x_{\omega} x_{0} x_{\omega^{2}} \delta_{g}$ & $-f(g) c_{4}$ & 0 & $c_{8}$ & 0 \\
$\quad-f(g) x_{1} x_{0} x_{\omega^{2}} \delta_{g}$ & & & & \\
$c_{9}=x_{1} x_{\omega} x_{0} x_{1} x_{\omega^{2}} \delta_{g}$ & $c_{5}$ & $c_{9}$ & 0 & 0 \\
$c_{10}=x_{0} x_{1} x_{0} x_{\omega} x_{0} x_{1} x_{\omega} x_{\omega^{2}} \delta_{g}$ & $-c_{6}$ & $-c_{10}$ & 0 & 0 \\
$\quad-f(g) x_{1} x_{\omega^{2}} \delta_{g}$ & & & & \\
$c_{11}=x_{\omega} x_{0} x_{1} x_{\omega^{2}} \delta_{g}$ & & & \\
$c_{12}=x_{1} x_{\omega} x_{0} x_{1} x_{\omega} x_{0} x_{\omega^{2}} \delta_{g}$ & & & \\
$\quad+x_{0} x_{1} x_{\omega} x_{0} x_{1} x_{\omega} x_{\omega^{2}} \delta_{g}-f(g) x_{\omega^{2}} \delta_{g}$ & & &
\end{tabular}


Table 4 Action of the generators $x_{i}$ on $L_{4}^{g}=\mathcal{A}_{G, \lambda} \mathbf{e}_{4}^{g}$

\begin{tabular}{l|c|c|c|c}
\hline \hline Linear basis of $L_{4}^{g}$ & $x_{0} \cdot$ & $x_{1} \cdot$ & $x_{\omega} \cdot$ & $x_{\omega^{2}}$ \\
\hline$c_{1}=x_{0} x_{1} x_{0} x_{\omega} x_{0} \delta_{g}$ & 0 & 0 & $-c_{6}$ & $-c_{10}$ \\
$c_{2}=x_{0} x_{1} x_{0} x_{\omega} x_{0} x_{1} x_{\omega} x_{0} \delta_{g}$ & 0 & 0 & $-f(g) c_{5}$ & $-c_{9}$ \\
$c_{3}=x_{0} x_{1} x_{\omega} x_{0} \delta_{g}-x_{0} x_{1} x_{0} x_{\omega^{2}} \delta_{g}$ & 0 & $c_{1}$ & $c_{12}$ & 0 \\
$c_{4}=x_{0} x_{1} x_{\omega} x_{0} x_{1} x_{\omega} x_{0} \delta_{g}-x_{0} x_{1} x_{0} x_{\omega} x_{0} x_{1} x_{\omega^{2}} \delta_{g}$ & 0 & $c_{2}$ & $c_{11}$ & 0 \\
$c_{5}=x_{0} x_{\omega} x_{0} \delta_{g}$ & 0 & $c_{7}$ & 0 & $-c_{3}$ \\
$c_{6}=x_{0} x_{\omega} x_{0} x_{1} x_{\omega} x_{0} \delta_{g}$ & 0 & $c_{8}$ & 0 & $-c_{4}$ \\
$c_{7}=x_{1} x_{0} x_{\omega} x_{0} \delta_{g}$ & $c_{2}$ & 0 & 0 & $-c_{12}$ \\
$c_{8}=x_{1} x_{0} x_{\omega} x_{0} x_{1} x_{\omega} x_{0} \delta_{g}$ & $c_{3}$ & 0 & $-c_{7}$ & $-c_{11}$ \\
$c_{9}=x_{1} x_{\omega} x_{0} \delta_{g}-x_{1} x_{0} x_{\omega^{2}} \delta_{g}$ & $c_{4}$ & 0 & $-c_{8}$ & 0 \\
$c_{10}=x_{1} x_{\omega} x_{0} x_{1} x_{\omega} x_{0} \delta_{g}-x_{1} x_{0} x_{\omega} x_{0} x_{1} x_{\omega^{2}} \delta_{g}$ & & & & \\
$\quad=f(g) \mathbf{e}_{4}^{g}$ & $c_{5}$ & $c_{9}$ & 0 & 0 \\
$c_{11}=x_{0} x_{1} x_{\omega} x_{0} x_{1} x_{\omega} x_{0} x_{\omega^{2}} \delta_{g}-f(g) x_{0} x_{\omega^{2}} \delta_{g}$ & & & & \\
$\quad+f(g) x_{\omega} x_{0} \delta_{g}$ & $c_{6}$ & $c_{10}$ & 0 & 0 \\
$c_{12}=-x_{0} x_{\omega} x_{0} x_{1} x_{\omega^{2}} \delta_{g}+x_{\omega} x_{0} x_{1} x_{\omega} x_{0} \delta_{g}$ & & &
\end{tabular}

Table 5 Action of the generators $x_{i}$ on $L_{5}^{g}=\mathcal{A}_{G, \lambda} \mathbf{e}_{5}^{g}$

\begin{tabular}{l|c|c|c|c}
\hline \hline Linear basis of $L_{5}^{g}$ & $x_{0} \cdot$ & $x_{1} \cdot$ & $x_{\omega} \cdot$ & $x_{\omega^{2}} \cdot$ \\
\hline$c_{1}=x_{0} x_{1} x_{0} x_{\omega} \delta_{g}$ & 0 & 0 & $-c_{6}$ & $c_{10}$ \\
$c_{2}=x_{0} x_{1} x_{0} x_{\omega} x_{0} x_{1} x_{\omega} \delta_{g}$ & 0 & 0 & $-c_{5}$ & $c_{9}$ \\
$c_{3}=x_{0} x_{1} x_{0} x_{\omega} x_{0} x_{1} x_{\omega} x_{0} x_{\omega^{2}} \delta_{g}$ & 0 & $f(g) c_{1}$ & $-f(g) c_{12}$ & 0 \\
& $+f(g) x_{0} x_{1} x_{\omega} \delta_{g}$ & & & \\
$c_{4}=x_{0} x_{1} x_{\omega} x_{0} x_{1} x_{\omega} \delta_{g}-x_{0} x_{1} x_{0} x_{\omega} x_{0} x_{\omega^{2}} \delta_{g}$ & 0 & $c_{2}$ & $c_{11}$ & 0 \\
& $=f(g) \mathbf{e}_{5}^{g}$ & & & \\
$c_{5}=x_{0} x_{1} x_{0} x_{\omega} x_{0} x_{1} x_{\omega} x_{0} \delta_{g}+f(g) x_{0} x_{\omega} \delta_{g}$ & 0 & $f(g) c_{7}$ & 0 & $c_{3}$ \\
$c_{6}=x_{0} x_{\omega} x_{0} x_{1} x_{\omega} \delta_{g}-f(g) x_{0} x_{\omega^{2}} \delta_{g}$ & 0 & $c_{8}$ & 0 & $c_{4}$ \\
$c_{7}=x_{1} x_{0} x_{\omega} \delta_{g}$ & $c_{1}$ & 0 & 0 & $c_{12}$ \\
$c_{8}=x_{1} x_{0} x_{\omega} x_{0} x_{1} x_{\omega} \delta_{g}$ & $c_{3}$ & 0 & $-f(g) c_{7}$ & 0 \\
$c_{9}=x_{1} x_{0} x_{\omega} x_{0} x_{1} x_{\omega} x_{0} x_{\omega^{2}} \delta_{g}+f(g) x_{1} x_{\omega} \delta_{g}$ & $c_{4}$ & 0 & $-c_{8}$ & 0 \\
$c_{10}=x_{1} x_{\omega} x_{0} x_{1} x_{\omega} \delta_{g}-x_{1} x_{0} x_{\omega} x_{0} x_{\omega} \delta_{g}$ & $c_{5}$ & $c_{9}$ & 0 & 0 \\
$c_{11}=x_{0} x_{\omega} x_{0} x_{1} x_{\omega} x_{0} x_{\omega} \delta_{g}$ & & & & \\
$\quad+x_{1} x_{0} x_{\omega} x_{0} x_{1} x_{\omega} x_{0} \delta_{g}+f(g) x_{\omega} \delta_{g}$ & $c_{6}$ & $c_{10}$ & 0 & 0 \\
$c_{12}=x_{\omega} x_{0} x_{1} x_{\omega} \delta_{g}-x_{0} x_{\omega} x_{0} x_{\omega^{2}} \delta_{g}$ & & & \\
\hline
\end{tabular}


Table 6 Action of the generators $x_{i}$ on $L_{6}^{g}=\mathcal{A}_{G, \lambda} \mathbf{e}_{6}^{g}$

\begin{tabular}{l|c|c|c|c}
\hline \hline Linear basis of $L_{6}^{g}$ & $x_{0} \cdot$ & $x_{1} \cdot$ & $x_{\omega} \cdot$ & $x_{\omega^{2}}$ \\
\hline$c_{1}=x_{0} x_{1} x_{0} \delta_{g}$ & 0 & 0 & $-c_{6}$ & $-c_{10}$ \\
$c_{2}=x_{0} x_{1} x_{0} x_{\omega^{2}} x_{0} x_{1} \delta_{g}$ & 0 & 0 & $-c_{5}$ & $c_{9}$ \\
$c_{3}=x_{0} x_{1} x_{0} x_{\omega} x_{0} x_{1} x_{\omega} x_{\omega^{2}} \delta_{g}+f(g) x_{0} x_{1} \delta_{g}$ & 0 & $f(g) c_{1}$ & $c_{12}$ & 0 \\
$c_{4}=x_{0} x_{1} x_{\omega} x_{0} x_{1} \delta_{g}-x_{0} x_{1} x_{0} x_{\omega} x_{\omega^{2}} \delta_{g}$ & 0 & $c_{2}$ & $c_{11}$ & 0 \\
$c_{5}=-x_{0} x_{1} x_{\omega} x_{0} x_{1} x_{\omega} x_{0} \delta_{g}+f(g) x_{0} \delta_{g}$ & 0 & $c_{7}$ & 0 & $c_{3}$ \\
$c_{6}=x_{0} x_{\omega} x_{0} x_{1} \delta_{g}$ & 0 & $c_{8}$ & 0 & $-c_{4}$ \\
$c_{7}=-x_{0} x_{1} x_{0} x_{\omega} x_{0} x_{1} x_{\omega} x_{0} \delta_{g}+f(g) x_{1} x_{0} \delta_{g}$ & $f(g) c_{1}$ & 0 & 0 & $c_{12}$ \\
$c_{8}=x_{1} x_{0} x_{\omega} x_{0} x_{1} \delta_{g}$ & $c_{2}$ & 0 & 0 & $c_{11}$ \\
$c_{9}=x_{1} x_{0} x_{\omega} x_{0} x_{1} x_{\omega} x_{0} \delta_{g}$ & 0 & $-c_{7}$ & 0 \\
$\quad-x_{0} x_{1} x_{0} x_{\omega} x_{0} x_{1} x_{\omega} \delta_{g}+f(g) x_{1} \delta_{g}$ & & & & \\
$c_{10}=x_{1} x_{\omega} x_{0} x_{1} \delta_{g}-x_{1} x_{0} x_{\omega} x_{\omega^{2}} \delta_{g}$ & $c_{4}$ & 0 & $-c_{8}$ & 0 \\
$c_{11}=x_{0} x_{\omega} x_{0} x_{1} x_{\omega} x_{\omega^{2}} \delta_{g}-x_{1} x_{\omega} x_{0} x_{1} x_{\omega} x_{0} \delta_{g}$ & $c_{5}$ & $c_{9}$ & 0 & 0 \\
$\quad-x_{0} x_{1} x_{\omega} x_{0} x_{1} x_{\omega} \delta_{g}+f(g) \delta_{g}=f(g) \mathbf{e}_{6}^{g}$ & & & & \\
$c_{12}=-x_{0} x_{1} x_{0} x_{\omega} x_{0} x_{1} x_{\omega} x_{0} x_{\omega^{2}} \delta_{g}$ & $f(g) c_{6}$ & $c_{10}$ & 0 & 0 \\
$\quad+f(g) x_{\omega} x_{0} x_{1} \delta_{g}-f(g) x_{0} x_{\omega} x_{\omega^{2}} \delta_{g}$ & & & & \\
\hline
\end{tabular}

Table 7 Weight of the vectors $c_{i}$ in the case $G^{\prime}=\mathbb{F}_{4} \rtimes C_{6}$

\begin{tabular}{c|c|c|c|c|c|c}
\hline \hline & $L_{1}^{g}$ & $L_{2}^{g}$ & $L_{3}^{g}$ & $L_{4}^{g}$ & $L_{5}^{g}$ & $L_{6}^{g}$ \\
\hline$c_{1}$ & $\left(0, t^{3}\right) g$ & $\left(\omega, t^{4}\right) g$ & $\left(0, t^{5}\right) g$ & $\left(\omega^{2}, t\right) g$ & $\left(\omega^{2}, t^{2}\right) g$ & $\left(\omega, t^{3}\right) g$ \\
$c_{2}$ & $g$ & $(\omega, t) g$ & $\left(0, t^{2}\right) g$ & $\left(\omega^{2}, t^{4}\right) g$ & $\left(\omega^{2}, t^{5}\right) g$ & $(\omega, 1) g$ \\
$c_{3}$ & $\left(1, t^{4}\right) g$ & $\left(\omega, t^{5}\right) g$ & $(1,1) g$ & $\left(0, t^{2}\right) g$ & $\left(0, t^{3}\right) g$ & $\left(\omega, t^{4}\right) g$ \\
$c_{4}$ & $(1, t) g$ & $\left(\omega, t^{2}\right) g$ & $\left(1, t^{3}\right) g$ & $\left(0, t^{5}\right) g$ & $g$ & $(\omega, t) g$ \\
$c_{5}$ & $\left(1, t^{5}\right) g$ & $g$ & $(1, t) g$ & $\left(\omega^{2}, t^{3}\right) g$ & $\left(\omega^{2}, t^{4}\right) g$ & $\left(0, t^{5}\right) g$ \\
$c_{6}$ & $\left(1, t^{2}\right) g$ & $\left(0, t^{3}\right) g$ & $\left(1, t^{4}\right) g$ & $\left(\omega^{2}, 1\right) g$ & $\left(\omega^{2}, t\right) g$ & $\left(0, t^{2}\right) g$ \\
$c_{7}$ & $\left(0, t^{4}\right) g$ & $\left(\omega^{2}, t^{5}\right) g$ & $g$ & $\left(1, t^{2}\right) g$ & $\left(1, t^{3}\right) g$ & $\left(\omega^{2}, t^{4}\right) g$ \\
$c_{8}$ & $(0, t) g$ & $\left(\omega^{2}, t^{2}\right) g$ & $\left(0, t^{3}\right) g$ & $\left(1, t^{5}\right) g$ & $(1,1) g$ & $\left(\omega^{2}, t\right) g$ \\
$c_{9}$ & $\left(\omega, t^{5}\right) g$ & $\left(\omega^{2}, 1\right) g$ & $(\omega, t) g$ & $\left(0, t^{3}\right) g$ & $\left(0, t^{4}\right) g$ & $\left(\omega^{2}, t^{5}\right) g$ \\
$c_{10}$ & $\left(\omega, t^{2}\right) g$ & $\left(\omega^{2}, t^{3}\right) g$ & $\left(\omega, t^{4}\right) g$ & $g$ & $(0, t) g$ & $\left(\omega^{2}, t^{2}\right) g$ \\
$c_{11}$ & $(\omega, 1) g$ & $(0, t) g$ & $\left(\omega, t^{2}\right) g$ & $\left(1, t^{4}\right) g$ & $\left(1, t^{5}\right) g$ & $g$ \\
$c_{12}$ & $\left(\omega, t^{3}\right) g$ & $\left(0, t^{4}\right) g$ & $\left(\omega, t^{5}\right) g$ & $(1, t) g$ & $\left(1, t^{2}\right) g$ & $\left(0, t^{3}\right) g$ \\
\hline
\end{tabular}

\section{Acknowledgments}

The authors thank professor Nicolás Andruskiewitsch for proposing this problem and useful suggestions for this article. The first author also thanks Carolina Renz for her hospitality during her stay in Córdoba.

Bárbara Pogorelsky was partially supported by Capes-Brazil. Cristian Vay was partially supported by ANPCyT-Foncyt, CONICET, MinCyT (Córdoba) and Secyt (UNC). 


\section{References}

[AAGMV] Andruskiewitsch, N., Angiono, I., García Iglesias, A., Masuoka, A. and VAY, C., Lifting via cocycle deformation. J. Pure Appl. Algebra, to appear. arXiv: $1212.5279 \mathrm{v} 1$.

[AAGTV] Andruskiewitsch, N., Angiono, I, Garcï£ia Iglesias, A., Torrecilla, B., VAY, C.: From Hopf algebras to tensor categories, aparecerá en Conformal field theories and tensor categories. Y.-Z. Huang, editor. Springer. arXiv:1204.5807v1.

[AG1] Andruskiewitsch, N., Graña, M., Braided Hopf algebras over non abelian finite groups, Bol. Acad. Ciencias (Córdoba) 63, 45-78, (1999).

[AG2] N. Andruskiewitsch and M. Graña, From racks to pointed Hopf algebras, Adv. Math. 178 (2003), $177-243$.

[AG3] N. AndruskiewitsCh and M. Graña, Examples of liftings of Nichols algebras over racks, AMA Algebra Montp. Announc. (electronic), Paper 1, (2003).

[An] Angiono, I., On Nichols algebras of diagonal type. J. Reine Angew. Math., to appear. arXiv: $1104.0268 \mathrm{v} 3$.

[AS] N. Andruskiewitsch and H.-J. Schneider, Pointed Hopf Algebras, in "New directions in Hopf algebras", 1-68, Math. Sci. Res. Inst. Publ. 43, Cambridge Univ. Press, Cambridge, 2002.

[AV1] Andruskiewitsch, N. and VAy, C. Finite dimensional Hopf algebras over the dual group algebra of the symmetric group in three letters, Commun. Algebra 39, 4507-4517, (2011).

[AV2] Andruskiewitsch, N. and VAY, C., On a family of Hopf algebras of dimension 72, Bull. Belg. Math. Soc. Simon Stevin 19, 415-443, (2012).

[BaW1] Barrett, J. W. and Westbury B. W. Spherical Categories. Adv. Math. 143 (1999), 357-375.

[BaW2] BARrett, J. W. , Invariants of piecewise-linear 3-manifolds. Trans. Amer. Math. Soc. 348 (1996), 3997-4022.

[CR] C. W. CuRTIS AND I. Reiner, Representation theory of finite groups and associative algebras. Reprint of the 1962 original. Wiley Classics Library. A WileyInterscience Publication. John Wiley \& Sons, Inc., New York, 1988. xiv+689 pp. ISBN: 0-471-60845-9.

[EG] Etingof, P. and Graña, M., On rack cohomology J. Pure Appl. Algebra 177, 49-59, (2003).

[ENO] Etingof, P., Nikshych, D. and Ostrik, V. On fusion categories. Ann. of Math. 162 (2005), 581-642.

[FMoS] Fischman, D; Montgomery, S; Schneider, H.-J., Frobenius extensions of subalgebras of Hopf algebras. Trans. Amer. Math. Soc. 349 (1997), no. 12, 48574895.

[G1] Graña, M., On Nichols algebras of low dimension, New trends on Hopf algebra theory (La Falda, 1999), Contemp. Math. 267 111-134, (2000).

[G2] M. GRAÑA, Zoo of finite-dimensional Nichols algebras of non-abelian group type, available at http://mate.dm.uba.ar/ matiasg/zoo.html.

[GIV] A. García IgLesias And C. VAY, Finite-dimensional pointed or copointed Hopf algebras over affine racks, J. Algebra, to appear. http://arxiv.org/abs/1210.6396v3

[HLV] Heckenberger, I., Lochmann, A., Vendramín, L., Braided racks, Hurwitz actions and Nichols algebras with many cubic relations, Transf. groups, to appear.

[J] Joyce, D., A classifying invariant of knots, the knot quandle, J. Pure Appl. Algebra 23, 37-65, (1982). Here is an account

[Mo] S. Montgomery, Hopf Algebras and their Actions on Rings, CBMS Reg. Conf. Ser. Math. 82, Amer. Math. Soc. (1993).

[NaVO] Nastasescu, C.; Van Oystaeyen, F., Methods of graded rings. Lecture Notes in Mathematics 1836. Springer-Verlag, Berlin, 2004.

[NeSc] Neunhöffer, M.; Scherotzke, S., Formulas for primitive idempotents in Frobenius algebras and an application to decomposition maps. Represent. Theory 12 (2008), 170-185.

[R] D. RAdFORD, Minimal quasitriangular Hopf algebras, J. Algebra 157 (1993), 285-315. 